\title{
A confocal study on the visualization of chromaffin cell secretory vesicles with fluorescent targeted probes and acidic dyes
}

\author{
Alfredo Moreno, Jaime SantoDomingo ${ }^{1}$, Rosalba I. Fonteriz, Carmen D. Lobatón, Mayte Montero, \\ Javier Alvarez*
}

Instituto de Biología y Genética Molecular (IBGM), Departamento de Bioquímica y Biología Molecular y Fisiología, Facultad de Medicina, Universidad de Valladolid and Consejo Superior de Investigaciones Científicas (CSIC), Ramón y Cajal, 7, E-47005 Valladolid, Spain

\section{A R T I C L E I N F O}

\section{Article history:}

Received 23 March 2010

Received in revised form 14 June 2010

Accepted 16 June 2010

Available online 22 June 2010

\section{Keywords:}

Confocal microscopy

Colocalization

Secretory granules

Chromaffin cells

Acidic dyes

Synaptobrevin 2

EGFP

\begin{abstract}
A B S T R A C T
Secretory vesicles have low pH and have been classically identified as those labelled by a series of acidic fluorescent dyes such as acridine orange or neutral red, which accumulate into the vesicles according to the $\mathrm{pH}$ gradient. More recently, several fusion proteins containing enhanced green fluorescent protein (EGFP) and targeted to the secretory vesicles have been engineered. Both targeted fluorescent proteins and acidic dyes have been used, separately or combined, to monitor the dynamics of secretory vesicle movements and their fusion with the plasma membrane. We have now investigated in detail the degree of colocalization of both types of probes using several fusion proteins targeted to the vesicles (synaptobrevin2-EGFP, Cromogranin A-EGFP and neuropeptide Y-EGFP) and several acidic dyes (acridine orange, neutral red and lysotracker red) in chromaffin cells, PC12 cells and $\mathrm{GH}_{3}$ cells. We find that all the acidic dyes labelled the same population of vesicles. However, that population was largely different from the one labelled by the targeted proteins, with very little colocalization among them, in all the cell types studied. Our data show that the vesicles containing the proteins more characteristic of the secretory vesicles are not labelled by the acidic dyes, and vice versa. Peptide glycyl-L-phenylalanine 2-naphthylamide (GPN) produced a rapid and selective disruption of the vesicles labelled by acidic dyes, suggesting that they could be mainly lysosomes. Therefore, these labelling techniques distinguish two clearly different sets of acidic vesicles in neuroendocrine cells. This finding should be taken into account whenever vesicle dynamics is studied using these techniques.
\end{abstract}

(c) 2010 Elsevier Inc. All rights reserved.

\section{Introduction}

Secretory vesicles have low $\mathrm{pH}$, around 5.5 , a property that share with other vesicular organelles such as the lysosomes. The low $\mathrm{pH}$ of all these vesicles should allow them to accumulate lipophilic compounds of acidic nature and, in fact, compounds with these characteristics such as acridine orange or neutral red label a vesicular population in neurons and neuroendocrine cells that has been classically assumed to correspond largely to the secretory vesicles (Kuijpers et al., 1989; Steyer et al., 1997; Steyer and Almers, 1999; Straub et al., 2000; Oheim and Stühmer, 2000). Thus, the dynamics of the vesicles labelled with acridine orange has been extensively investigated to monitor vesicle motion, fusion with the plasma membrane and other characteristics of the latter steps be-

\footnotetext{
Abbreviations: EGFP, enhanced green fluorescent protein; NPY, neuropeptide Y; VAMP, vesicle-associated membrane protein; FCCP, carbonyl cyanide 4-(trifluoromethoxy)phenylhydrazone; GPN, glycyl-L-phenylalanine 2-naphthylamide.

* Corresponding author. Fax: +34 983423588.

E-mail address: jalvarez@ibgm.uva.es (J. Alvarez).

${ }^{1}$ Present address: Department of Cell Physiology and Metabolism, University of Geneva, 1 rue Michel Servet, CH-1211 Geneva 4, Switzerland.
}

fore fusion (Steyer et al., 1997; Steyer and Almers, 1999; Oheim and Stühmer, 2000; Li et al., 2004).

More recently, several chimeric proteins targeted to the secretory vesicles and containing EGFP have been engineered and expressed in different cells (Lang et al., 1997; Tsuboi et al., 2000; Ohara-Imaizumi et al. 2002; Bezzi et al., 2004; Allersma et al., 2004, 2006), and used also to investigate the dynamics of the secretory vesicles. In some cases, cells expressing one of these constructs were also labelled with acridine orange to monitor at the same time the dynamics of the vesicles, using the specifically targeted EGFP marker, and the event of fusion, by following the disappearance of the loaded dye (Tsuboi et al., 2000; Bezzi et al., 2004). These papers showed an extensive colocalization among the two types of probe. However, it has been reported more recently that acridine orange metachromasie, that results in the concomitant emission of green and red fluorescence from acridine orange, generates systematic colocalization errors between acridine orange and EGFP in vesicular organelles (Nadrigny et al., 2007). According to this work, the green emission from acridine orange overlaps with that of EGFP and produces a false apparent colocalization on dual-color images. 
We have now made a detailed study of the colocalization of several EGFP-probes targeted to the secretory vesicles and several acidic dyes. Our results show that both kinds of labelling methods produce a clear vesicular pattern, but surprisingly there was little coincidence among the vesicular patterns generated using EGFPprobes and those obtained with acidic dyes.

\section{Materials and methods}

\subsection{Preparation and culture of chromaffin cells, $\mathrm{PC1} 2$ cells and $\mathrm{GH}_{3}$ cells}

Ethical approval for this study was granted from the investigation committee and the animal experimentation committee of the Faculty of Medicine, University of Valladolid. Cow adrenal glands were kindly supplied by the veterinaries of the slaughterhouse Justino Gutiérrez of Laguna de Duero (Valladolid). Bovine adrenal medulla chromaffin cells were isolated as described previously (Moro et al., 1990), plated on $12 \mathrm{~mm}$ glass polilysine-coated coverslips $\left(0.25 \times 10^{6}\right.$ cells per $1 \mathrm{ml}$ medium $)$ and cultured in high-glucose $(4.5 \mathrm{~g} / \mathrm{l})$ Dulbecco's modified Eagle medium (DMEM) supplemented with $10 \%$ fetal bovine serum, 50iu ml ${ }^{-1}$ penicillin and 50iu ml ${ }^{-1}$ streptomycin. Cultures were maintained at $37^{\circ} \mathrm{C}$ in a humidified atmosphere of $5 \% \mathrm{CO}_{2}$. PC12 rat pheochromocytoma cells were grown in high-glucose $(4.5 \mathrm{~g} / \mathrm{l})$ Dulbecco's modified Eagle's medium supplemented with $7.5 \%$ fetal calf serum, $7.5 \%$ horse serum and $2 \mathrm{mM}$ glutamine. $\mathrm{GH}_{3}$ adenohypophyseal cells were grown in RPMI 1640 culture medium supplemented with $2.5 \%$ fetal bovine serum, $15 \%$ horse serum, $2 \mathrm{mM}$ glutamine, $100 \mathrm{iu} \mathrm{ml} \mathrm{m}^{-1}$ penicillin and 100 iu ml ${ }^{-1}$ streptomycin at $37{ }^{\circ} \mathrm{C}$ in a humidified atmosphere of $5 \% \mathrm{CO}_{2}$. Cells were seeded over glass bottom Petry dishes coated with poly-L-lisine $(0.01 \mathrm{mg} / \mathrm{ml})$.

\subsection{Preparation and expression of the EGFP-targeted probes}

The VAMP-enhanced green fluorescent protein (EGFP) construct has been described previously (SantoDomingo et al., 2008). For construction of adenoviral vectors, full-length cDNA encoding these constructs was subcloned into the pShuttle vector and then used for construction of the corresponding adenoviral vector by using an AdenoX adenovirus construction kit (Clontech). Cells were infected with an adenovirus for expression of this construct. Infection was carried out the day after cell isolation and $\mathrm{Ca}^{2+}$ measurements were performed $48-72 \mathrm{~h}$ after infection. Efficiency of infection of chromaffin cells with the adenovirus carrying the VAMP-EGFP chimera was estimated to be about $60 \%$.

The chromogranin A-EGFP and neuropeptide Y-EGFP constructs were kindly provided by Dr. J.D. Machado, University of La Laguna, Spain. Transfections of these constructs were carried out using Metafectene (Biontex, Germany).

\subsection{Confocal studies}

Cells were imaged at room temperature on a Leica TCS SP2 confocal spectrophotometer using a $63 \times$ oil immersion objective. EGFP-containing constructs and acridine orange were excited with the $488 \mathrm{~nm}$ line of the Argon laser, and the fluorescence emitted between 500 and $530 \mathrm{~nm}$ was collected. Fluorescence from lysotracker red or neutral red dyes was excited with the $543 \mathrm{~nm}$ line of the green He-Ne laser and the fluorescence emitted between 600 and $700 \mathrm{~nm}$ was collected. The above settings were carefully chosen to assure that there was no interference from the green fluorochrome in the red channel, or viceversa. Lack of bleedthrough between the two channels can be clearly appreciated in many of the figures. Images for each fluorochrome at every confo- cal plane were recorded sequentially frame by frame at a rate of 0.8 frames per second. No significant movement of the granules was observed when consecutive images of the same fluorophore were taken at this rate. For loading with the acidic dyes, cells were incubated for 1-5 min with either $100 \mathrm{nM}$ acridine orange, $50 \mathrm{nM}$ lysotracker red or $1 \mu \mathrm{M}$ neutral red, added directly to the cell chamber in the stage of the microscope.

For colocalization analysis we have used the toolbox JACoP (Bolte and Cordelières, 2006) under ImageJ software (public domain image processing program developed by Wayne Rasband at the National Institutes of Health, Bethesda, U.S.A.) to obtain the Pearson's correlation coefficients (Manders et al., 1992) from deconvolved images of each channel. When this coefficient that can vary between -1 and +1 is applied to image colocalization, values close to +1 indicate colocalization, while values close to zero indicate lack of correlation. The values obtained in each case are given in the Figure Legends. In Fig. 1A, the composite images showing the colocalized pixels were obtained with the Colocalization Finder plugin from the Image software.

\subsection{Fluorescence microscopy measurements}

Cells expressing VAMP-EGFP were mounted in a cell chamber in the stage of a Zeiss Axiovert 200 microscope under continuous perfusion. Single cell fluorescence was excited at $480 \mathrm{~nm}$ using a Cairn monochromator (200 ms excitation every $2 \mathrm{~s}, 10 \mathrm{~nm}$ bandwidth) and images of the emitted fluorescence obtained with a $40 \times$ Fluar objective were collected using a 495DCLP dichroic mirror and a E515LPV2 emission filter (both from Chroma Technology) and recorded by a Hamamatsu ORCA-ER camera. Single cell fluorescence records were analyzed using the Metafluor program (Universal Imaging). Experiments were performed at room temperature.

\section{Results}

3.1. Subcellular dual-color localization of VAMP-EGFP and acidic dyes: lysotracker red and neutral red

Given that VAMP-EGFP and acridine orange fluorescences cannot be well distinguished, we have used other two acidic dyes having a fluorescence spectrum that can be easily separated from that of EGFP by choosing the appropriate emission windows, as described in Methods: lysotracker red and neutral red. Fig. 1A and B show a series of confocal images of two chromaffin cells expressing VAMP-EGFP and then stained with lysotracker red. It can be observed that both VAMP-EGFP and lysotracker red generated a vesicular pattern. In addition, VAMP-EGFP also labelled the plasma membrane. This was expected, as it is an integral protein of the vesicle membrane and remains in the plasma membrane after fusion. However, the vesicular patterns observed with both probes were clearly different and mostly non-coincident. Because yellow pixels are sometimes difficult to see over the red and green background, a series of images showing in bright white the few coincident pixels has been included in Fig. 1A to make clear that the coincidence is marginal. Accordingly, Pearsońs correlation coefficients were also close to zero (see legend). In addition to the lack of colocalization, vesicle distribution and size was very different in both groups: vesicles stained with lysotracker red were less in number and generally bigger than those labelled by VAMP-EGFP.

Similar findings were observed in $\mathrm{PC} 12$ and $\mathrm{GH}_{3}$ cells. Fig. 2 shows confocal planes of each of these cells expressing VAMPEGFP and then stained with lysotracker red. Although it is difficult to exclude some small degree of colocalization, in part due to the large density of vesicles labelled by VAMP-EGFP, it is clear that the vesicular patterns in both cases are completely different, and 

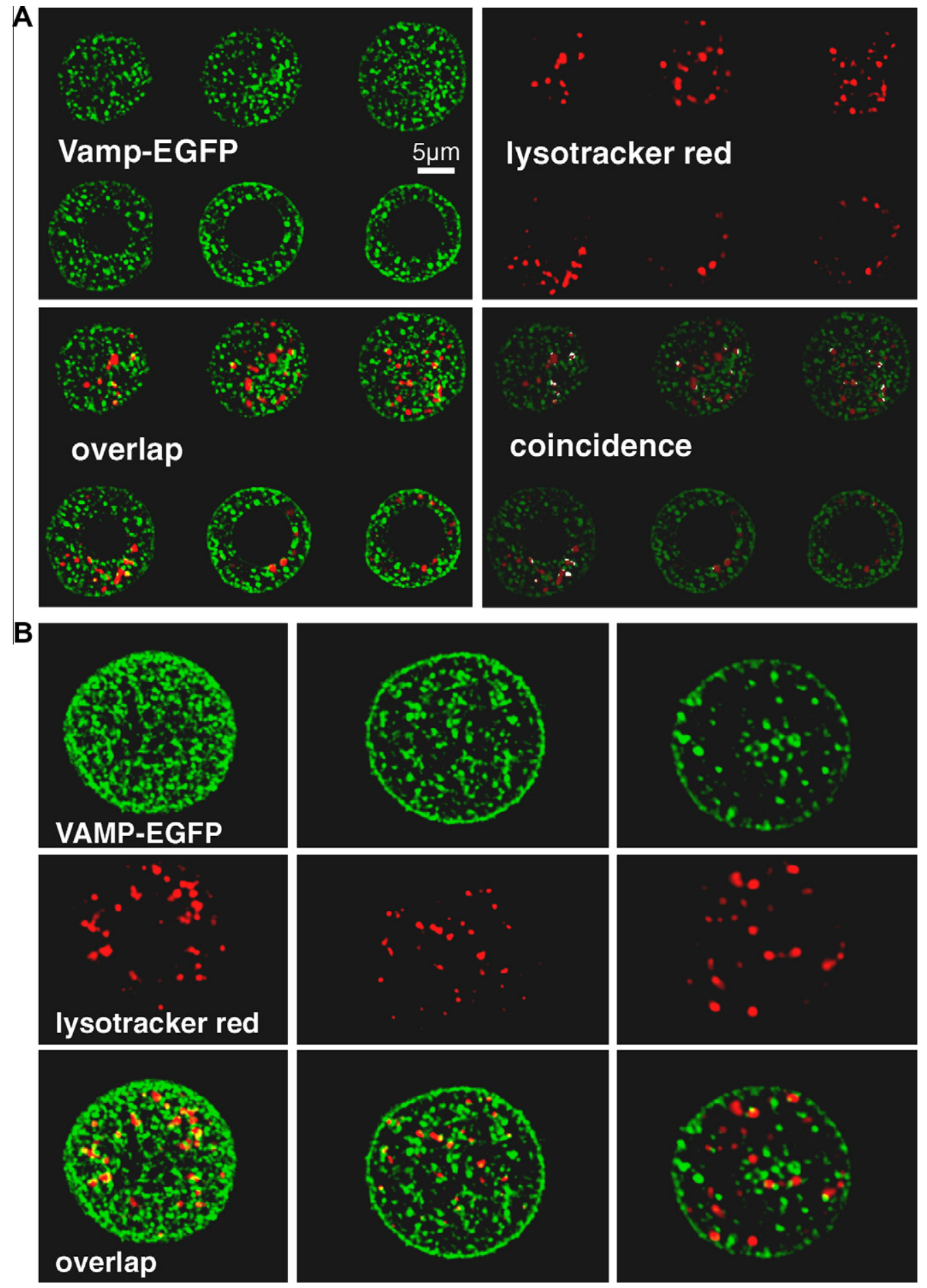

Fig. 1. Confocal colocalization study of VAMP-EGFP and lysotracker red fluorescence in bovine chromaffin cells. Panel A shows images obtained in six different planes of a single bovine chromaffin cell and panel B shows images obtained in three different planes of another cell. The green images show in both panels the fluorescence obtained in cells expressing VAMP-EGFP using the $488 \mathrm{~nm}$ excitation line of the Ar laser and monitoring the fluorescence emitted between 500 and $530 \mathrm{~nm}$. The red images show the fluorescence emitted by the same cells in the same confocal plane between 600 and $700 \mathrm{~nm}$ after loading with $50 \mathrm{nM}$ lysotracker red for $1 \mathrm{~min}$ and using the $543 \mathrm{~nm}$ excitation line of the green He-Ne laser. The overlap images show the superimposition of both fluorescences. The coincidence images in panel A have been obtained with the Colocalization Finder plugin from the ImageJ software and show in bright white the colocalized pixels. Pearsońs coefficients corresponding to all the colocalizations ranged between 0.028 and 0.110 . Data are representative of about 100 similar cells studied.

this is confirmed by the very small Pearsońs correlation coefficients obtained.

Fig. 3 shows a confocal image of a chromaffin cell expressing VAMP-EGFP and then stained with a different acidic dye, neutral red. The images are very similar to those obtained previously in cells labelled with both VAMP-EGFP and lysotracker red. Neutral red also labelled here a smaller number of large-size vesicles, which were little coincident with those expressing VAMP-EGFP. 

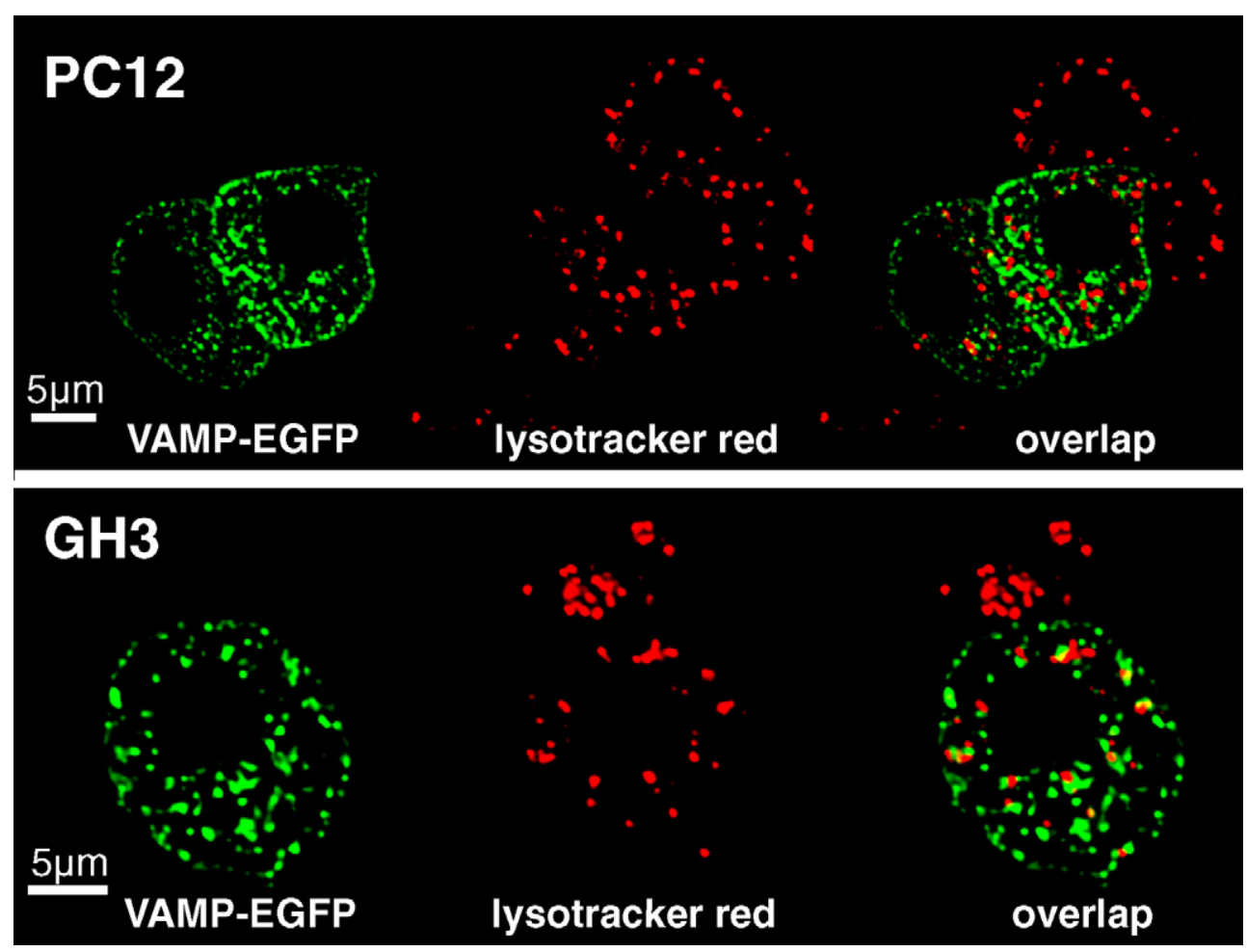

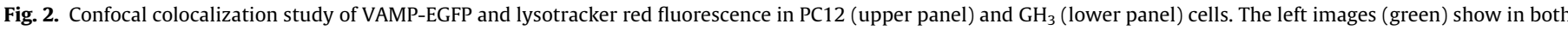

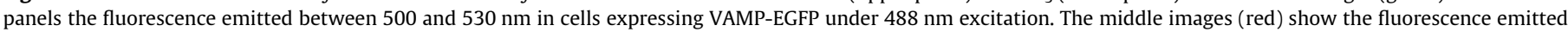

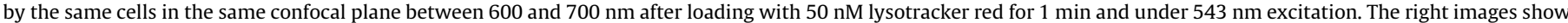

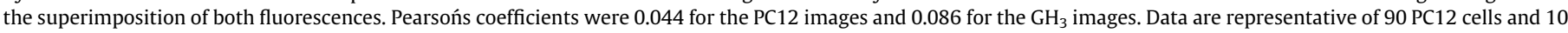
$\mathrm{GH}_{3}$ cells studied.

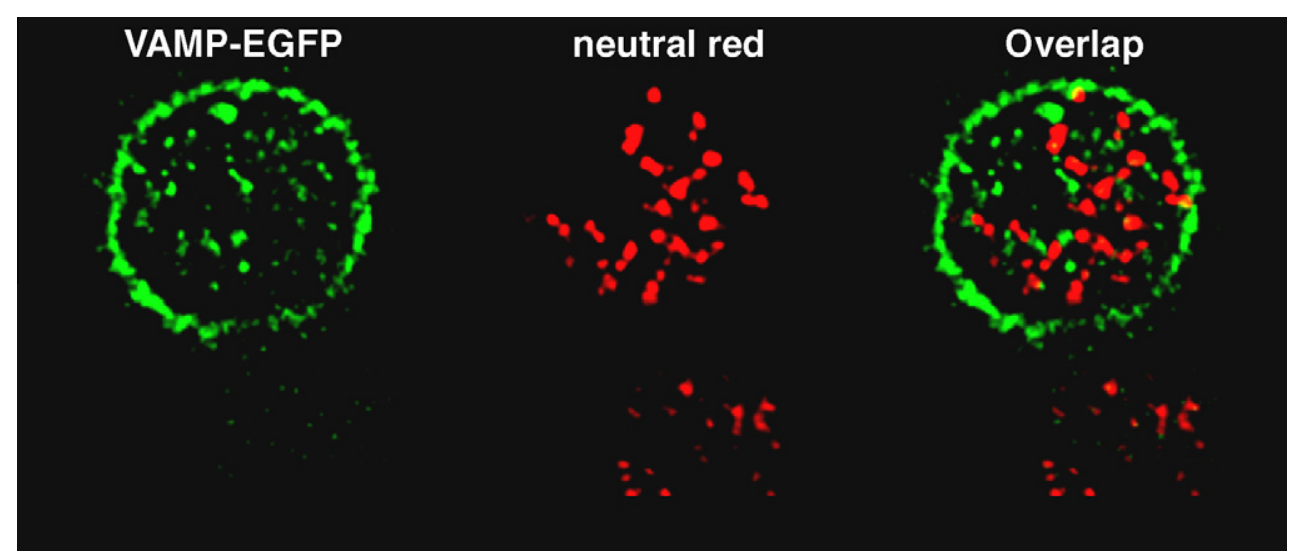

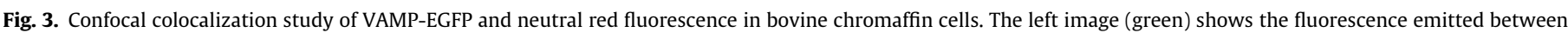

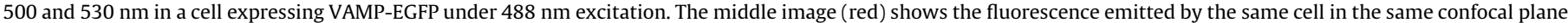

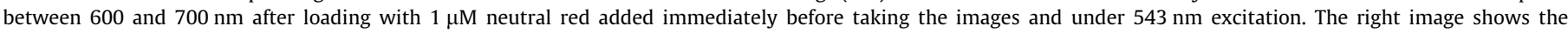
superimposition of both fluorescences. Pearsońs coefficient was 0.030 . Data are representative of 12 similar cells studied.

\subsection{Colocalization of acridine orange with other acidic dyes}

As mentioned above, colocalization of VAMP-EGFP and acridine orange is difficult to study. However, the fluorescence of acridine orange can be easily separated from that of lysotracker red or neutral red. Given that we know that these dyes do not colocalize with VAMP-EGFP, studying the colocalization of acridine orange with these dyes can provide us clues on the colocalization of acridine orange and VAMP-EGFP. Fig. 4A shows a confocal image of a PC12 cell stained with both acridine orange and lysotracker red, and it can be seen that both fluorescences colocalize extensively. The same hap- pens when the cells are stained with both acridine orange and neutral red, as shown in Fig. 4B. In both cases, Pearsońs coefficients were close to the unity (see the legend), confirming the colocalization of both signals. Therefore, acridine orange labels the same vesicular compartment labelled by lysotracker red or neutral red.

We wanted to test also if the colocalization among acridine orange and lysotracker red could be also seen in cells expressing VAMP-EGFP. That was the case. Fig. 5A shows PC12 cells expressing VAMP-EGFP and then stained with lysotracker red. As shown above, the overlap shows that there was little colocalization among both fluorescences. Accordingly, Pearsońs coefficient was 

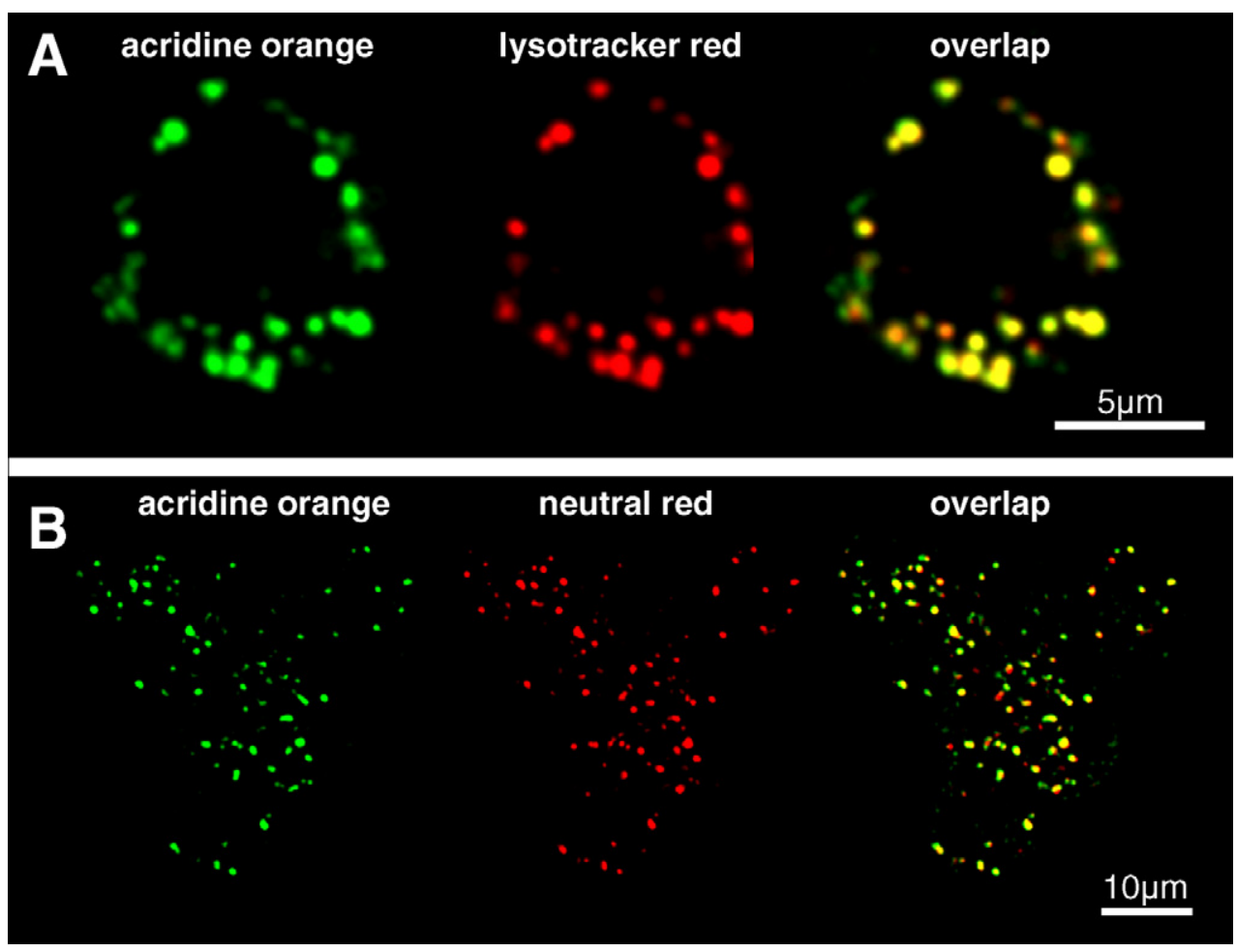

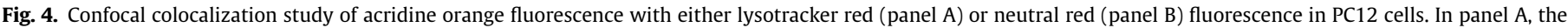

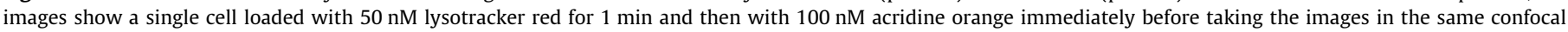

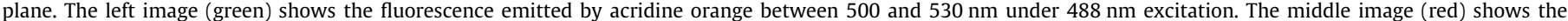

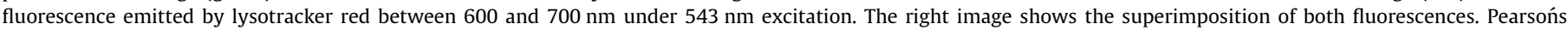

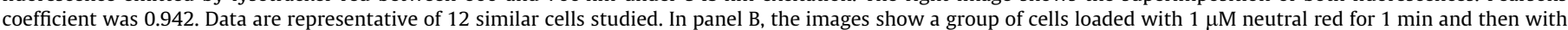

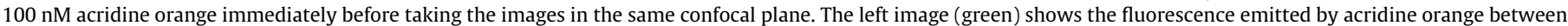

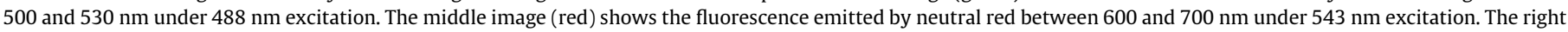
image shows the superimposition of both fluorescences. Pearsońs coefficient was 0.764 . Data are representative of eight similar cells studied.

very small, 0.122 . Then, Fig. $5 \mathrm{~B}$ shows the result of labelling the same cells of Fig. 5A with acridine orange. Now the left image (green) shows the fluorescences of both VAMP-EGFP and acridine orange observed together in the same channel. The middle image (red) shows the fluorescence of lysotracker red, and the right image shows the superimposition. The images of lysotracker red slightly differ among panels $A$ and $B$ because of vesicle movement or changes in focus during the time required for acridine orange loading. As expected, there was an increase in the degree of colocalization of red and green fluorescences. Pearsońs coefficient increased to 0.313 . Of course, colocalization is not complete because of the lack of red counterpart for the VAMP-EGFP fluorescence.

\subsection{Colocalization of lysotracker red with either chromogranin A-EGFP or NPY-EGFP}

We have then tested if the same findings obtained with VAMPEGFP could also be obtained using other methods to target EGFP to the vesicles. Fig. 6 shows confocal images of PC12 cells expressing either chromogranin A-EGFP (panel A) or NPY-EGFP (panel B) and then stained with lysotracker red. We can see essentially the same findings obtained previously with VAMP-EGFP. Again, the EGFP fluorescence shows a large number of small vesicles (now there is no fluorescence in the plasma membrane, as EGFP is fused to soluble proteins). Instead, lysotracker red labels a smaller number of vesicles with a larger size, that show little colocalization with those labelled by the EGFP-targeted constructs.
3.4. Absence of colocalization of VAMP-EGFP and lysotracker red after prolonged expression of VAMP-EGFP

It could be argued that the transient expression of any of the targeted EGFP-containing proteins after transfection or infection could lead to only a partial labelling of the vesicular compartment, due to the time required for vesicle maturation. To avoid this problem, we have generated PC12 cells expressing VAMP-EGFP for prolonged periods (up to 15 days). These cells are continuously producing the protein, so that it should be able to label the vesicles in all the states of maturation. Fig. 7 shows the fluorescence of VAMP-EGFP in these cells, together with that of lysotracker red and the superposition. Again here, both types of labelling showed a very different vesicular pattern, as observed before, and Pearsońs coefficients remain low, 0.171 .

In conclusion, our data show that all the acidic dyes, including lysotracker red, neutral red and acridine orange, labelled in several neuroendocrine cells a vesicular compartment that was largely different from the one labelled with the targeted proteins. The reason was not that VAMP-EGFP was in a non-acidic vesicular compartment. Fig. 8 shows that, as has been reported before (Camacho et al., 2006), vesicle alkalinization with the protonophore carbonyl cyanide 4-(trifluoromethoxy)phenylhydrazone (FCCP) induced a large increase in VAMP-EGFP fluorescence, showing that VAMPEGFP is actually present in an acidic compartment. Regarding the nature of the compartment labelled by acidic dyes, it could probably be assigned to lysosomes or endosomes. To investigate this hypothesis, we have tested the effect of the peptide glycyl-L-phenylalanine 2-naphthylamide (GPN) on cells doubly-stained with 

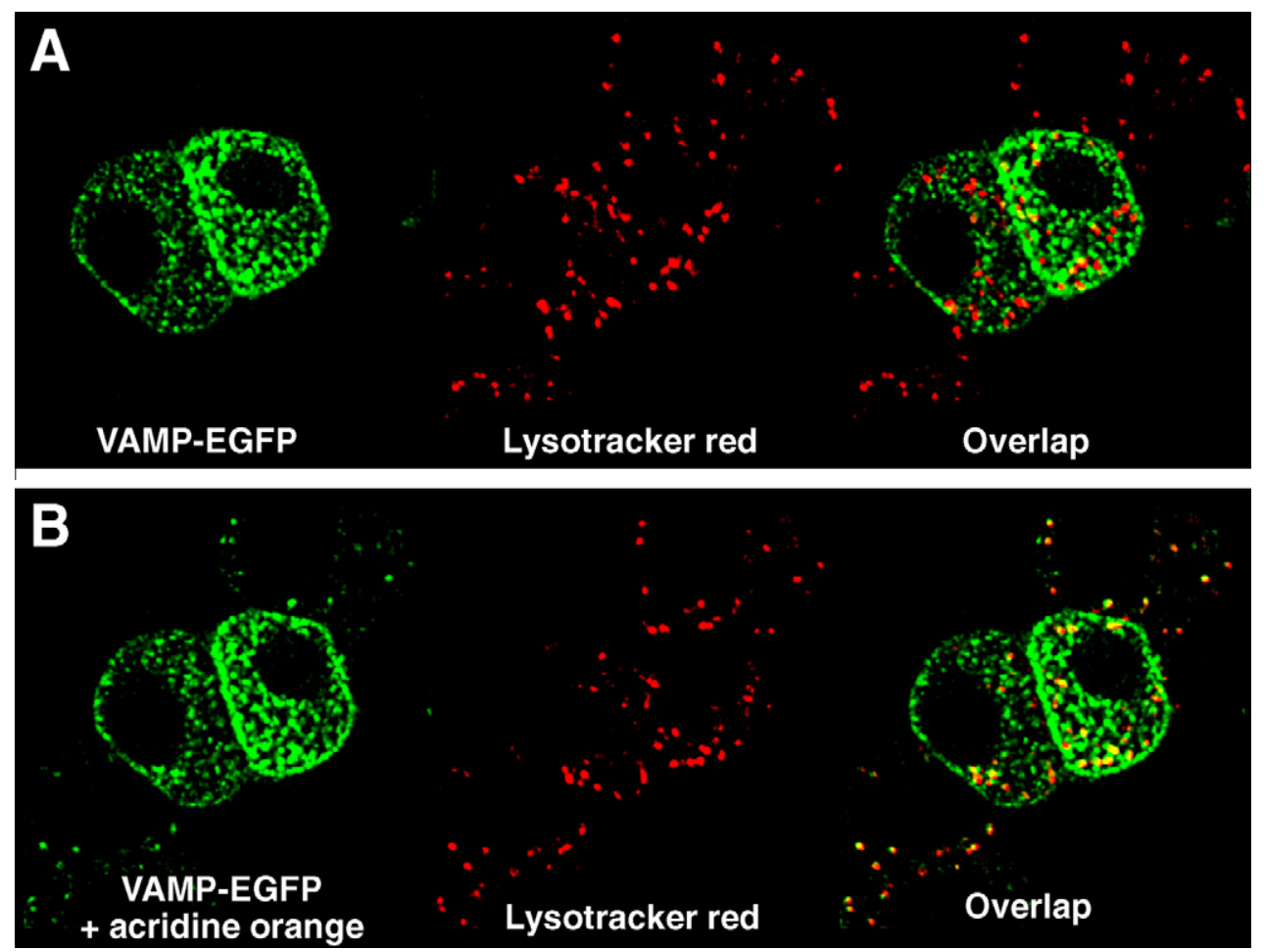

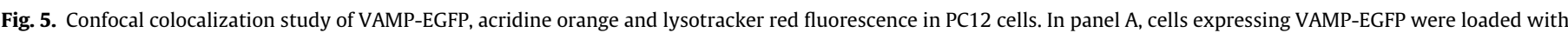

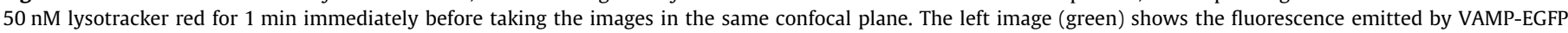

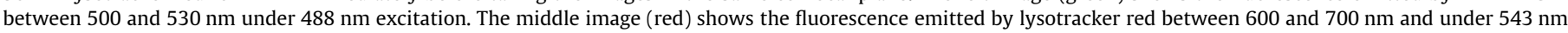

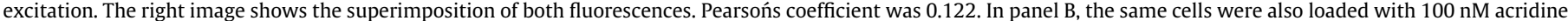

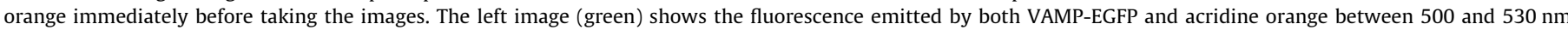

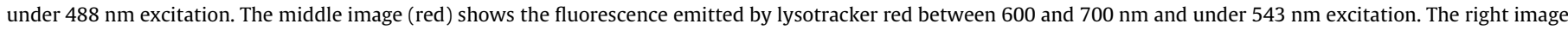
shows the superimposition of both fluorescences. Pearsońs coefficient was 0.313 . Data are representative of 12 similar cells studied.
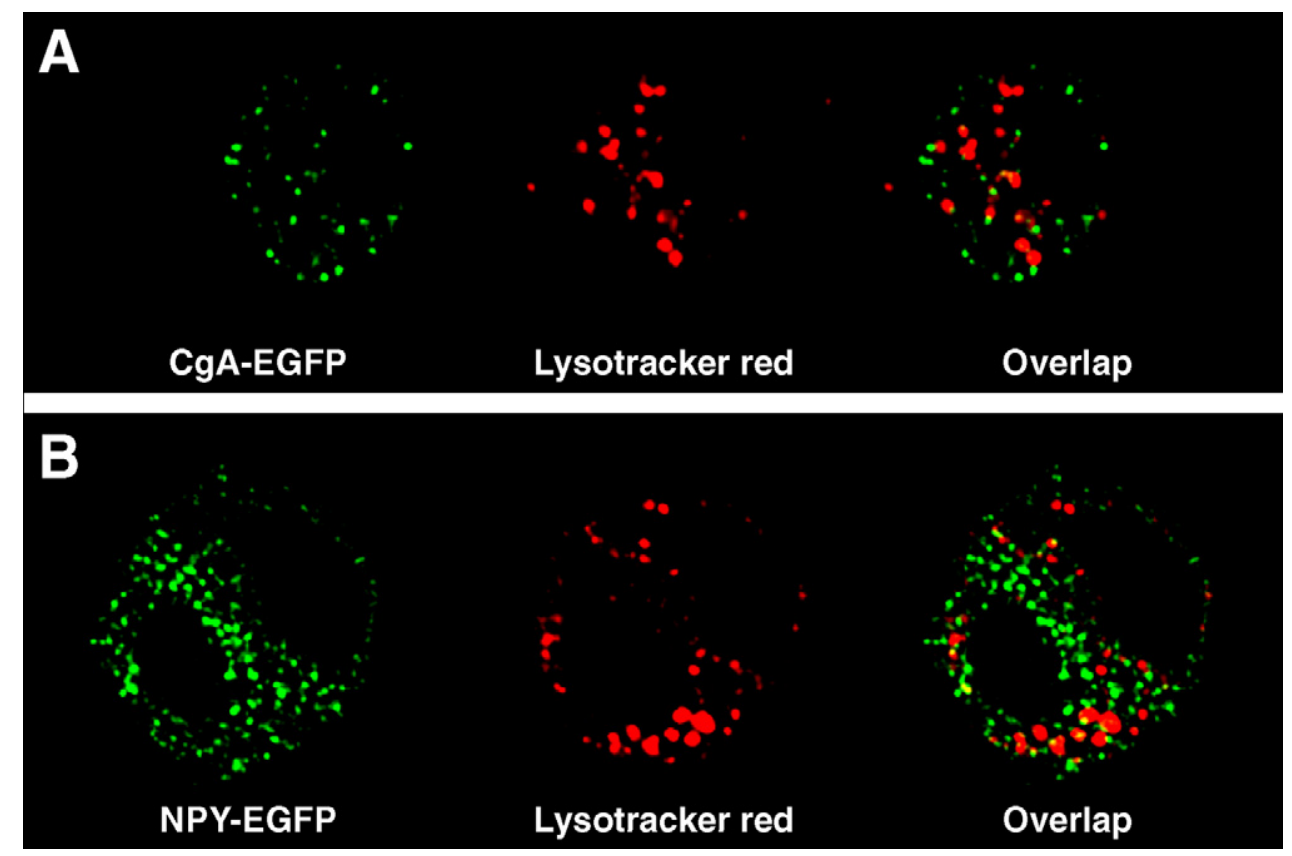

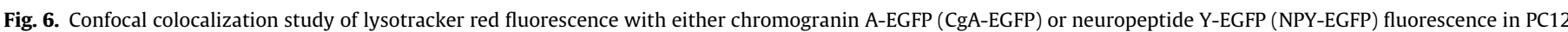

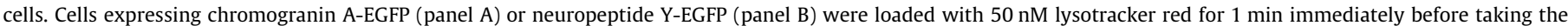

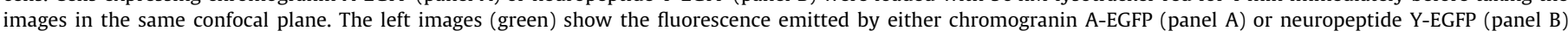

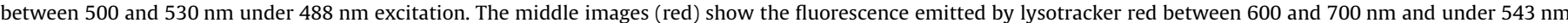

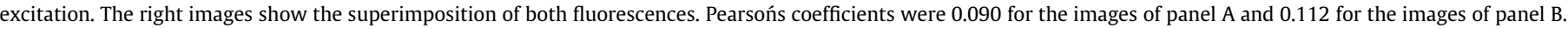
Data are representative of 10 cells expressing chromogranin-EGFP and 15 cells expressing neuropeptide Y-EGFP studied. 


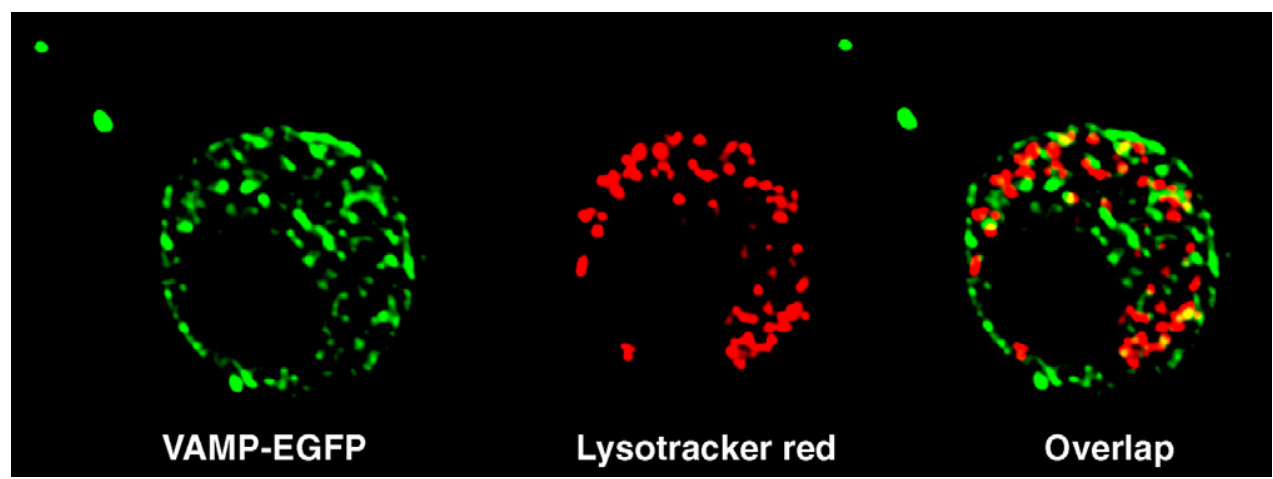

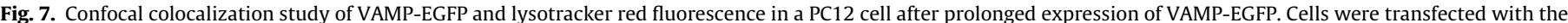

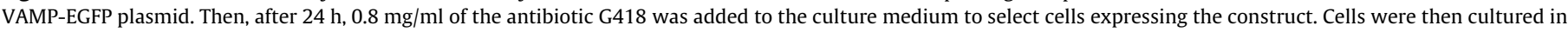

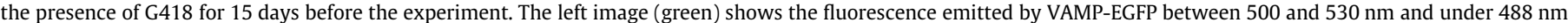

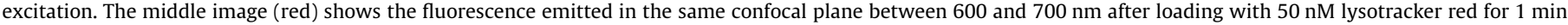

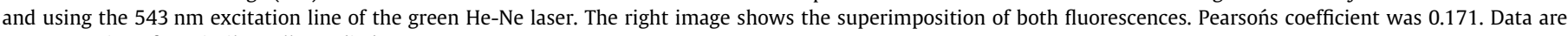
representative of 10 similar cells studied.

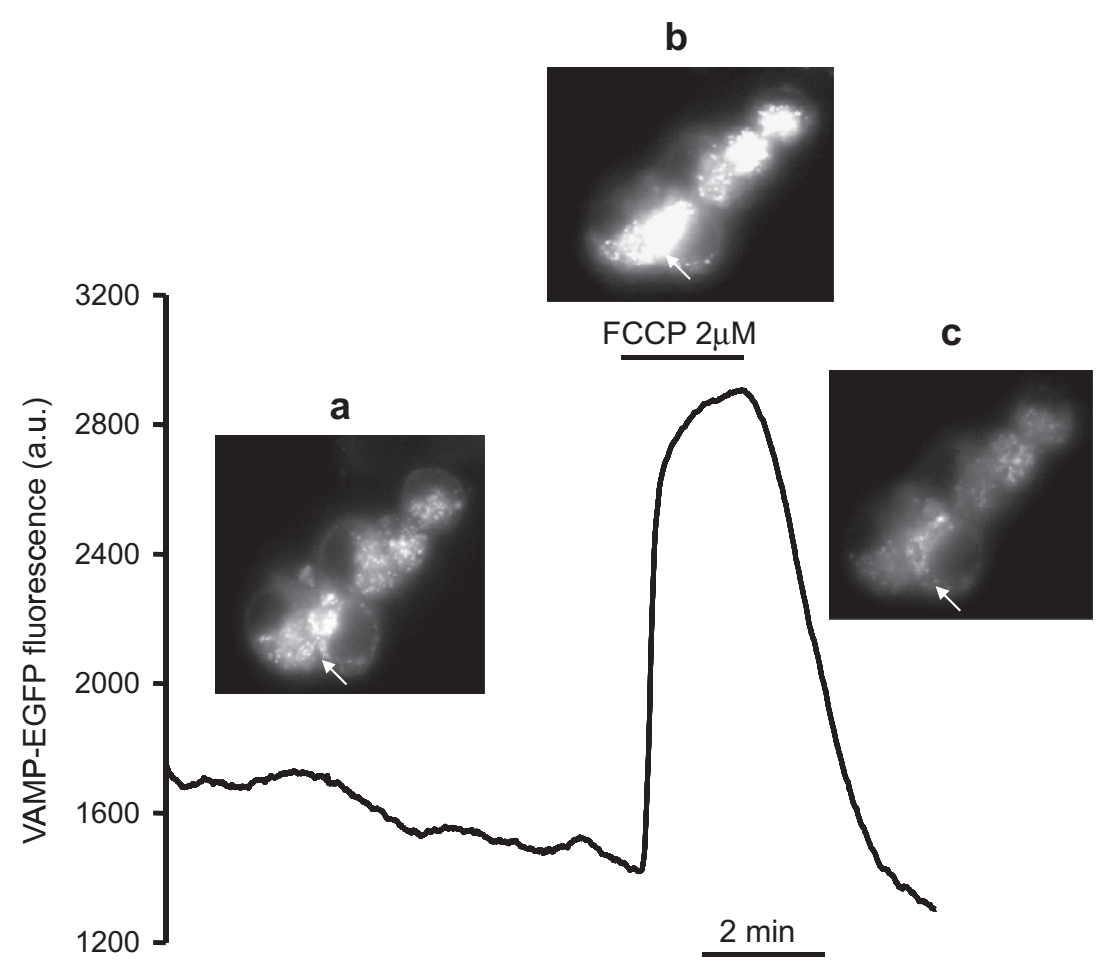

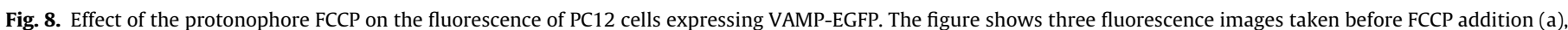

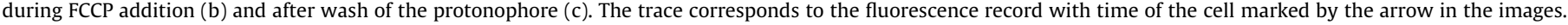
Data are representative of 4 similar experiments.

VAMP-EGFP and lysotracker red. This peptide has been reported to selectively permeabilize lysosomes (Jadot et al., 1990; Haller et al., 1996), although effects on other subcellular organelles have also been described (Duman et al., 2006). In agreement with our hypothesis, the peptide induced a fast disappearance of the lysotracker red fluorescence. Fig. 9 shows the confocal images of EGFP-VAMP and lysotracker red fluorescences before and $2 \mathrm{~min}$ after the addition of $0.5 \mathrm{mM}$ GPN. It can be observed that GPN induced a fast and nearly complete disappearance of the lysotracker red fluorescence, while the green EGFP-VAMP one remained intact or became even slightly more intense.

\section{Discussion}

We have used several EGFP-probes targeted to the secretory vesicles and several acidic dyes to investigate the degree of colocalization among both types of probes. Our data show that all of these probes label a vesicle population in several neuroendocrine cells, but the populations labelled by the targeted proteins and the dyes were largely different. Although protein overexpression may sometimes alter their pattern of intracellular distribution, this is probably not the case here because this phenomenon would normally increase the degree of colocalization. In addition, images 

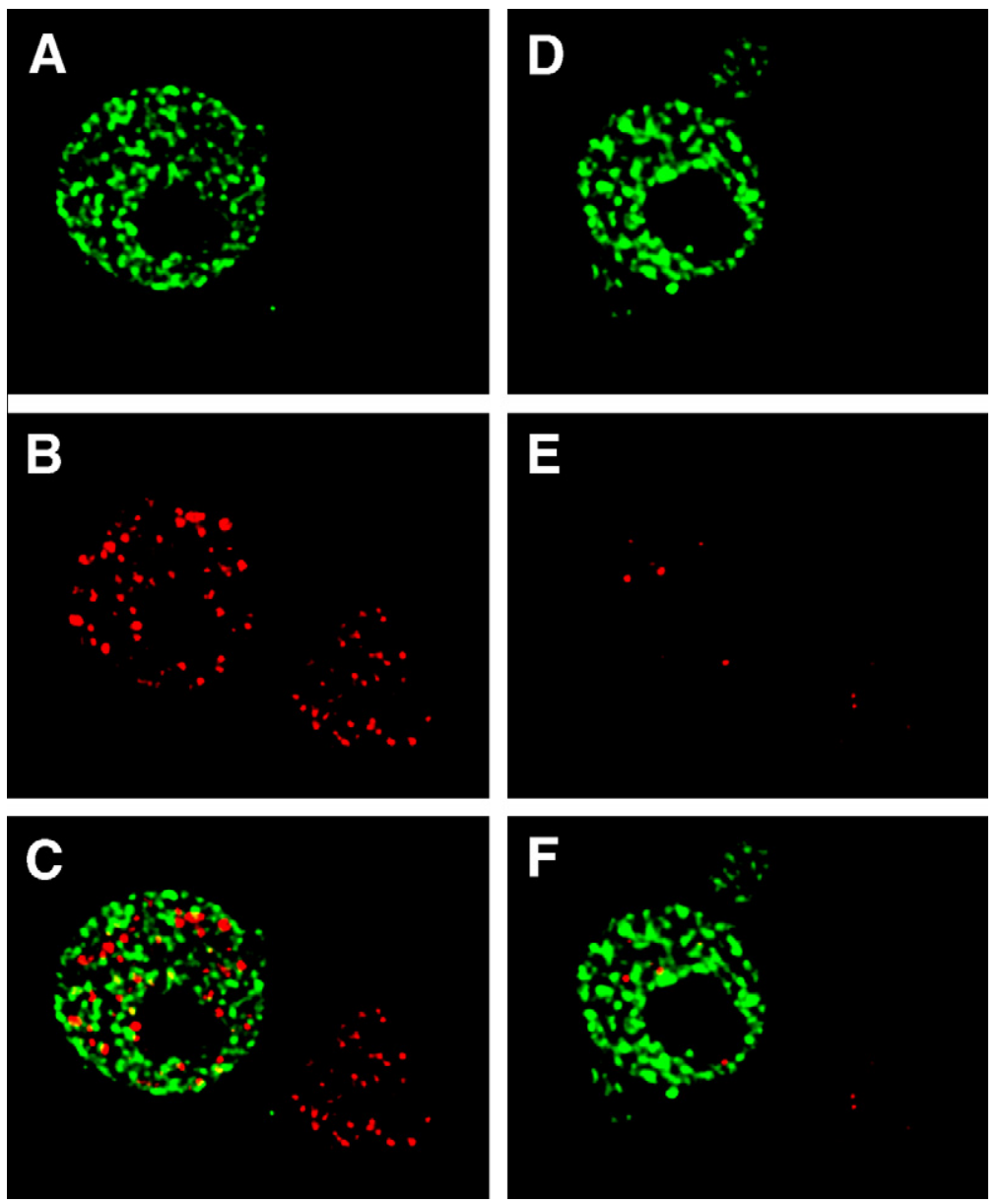

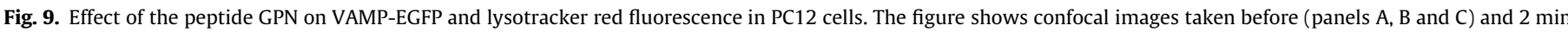

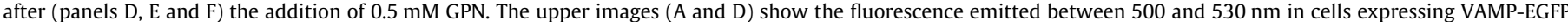

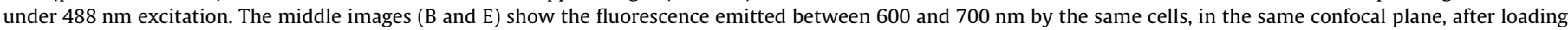

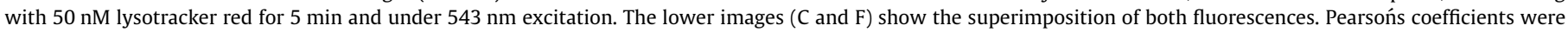
0.125 for the left image and 0.020 for the right image. Data are representative of 15 cells studied.

taken in cells with different levels of EGFP-targeted proteins expression (see Figs. 2, 5 and 6) showed also a similar lack of colocalization with the acidic dyes.

Our data contrast with previous data of several authors showing colocalization of EGFP-targeted probes with either acridine orange (Tsuboi et al., 2000; Bezzi et al., 2004) or lysotracker red (Duncan et al. 2003). Regarding the colocalization with acridine orange, it has been reported more recently the presence of systematic colocalization errors in vesicular organelles due to the presence of both red and green emission from acridine orange (Nadrigny et al., 2007), that could explain the discrepancy. Regarding the colocalization of EGFP-atrial natriuretic factor with lysotracker red reported by Duncan et al. (2003), the origin of the discrepancy is more difficult to find. In that paper the EGFP-targeted probe was reported to colocalize $96 \%$ with lysotracker red, while only $1 \%$ of lysotracker red colocalized with the green EGFP fluorescence. This implies that there should be 100 -fold more vesicles labelled with lysotracker red than with the EGFP-targeted probe. Our data and also data from other authors using acridine orange are not consistent with such a larger amount of vesicles labelled with the acidic dye with respect to those labelled with the EGFP-targeted probe.

The selective and nearly complete disruption by GPN of the vesicles labelled by acidic dyes suggests that these vesicles correspond mainly to lysosomes. The reason by which the acidic dyes do not label also most of the secretory granules expressing the specific targeting proteins is obscure. They have low $\mathrm{pH}$, about 5.5, and therefore their $\mathrm{pH}$ is not very different from that of lysosomes or endosomes. We can only speculate on the high viscosity of the granule matrix of the large dense-core vesicles, which could quench the fluorescence or perhaps even reduce loading. Whatever may be the reason; our data indicate that EGFP-targeted probes are much more adequate to study the behavior of the secretory vesicles than acidic dyes.

\section{Conclusions}

Our data show that there are two types of acidic vesicles in neuroendocrine cells which can be easily distinguished by confocal microscopy. Those containing the proteins more characteristic of the secretory granules, such as VAMP, chromogranin A or NPY, were labelled using EGFP-containing targeted chimeric proteins (VAMP-EGFP, chromogranin A-EGFP or NPY-EGFP) but not with acidic dyes. Instead, the vesicles labelled with acidic dyes showed little labelling with the targeted chimeras.

\section{Acknowledgments}

This work was supported by grants from Ministerio de Educación y Ciencia (BFU2008-01871) and from Junta de Castilla y León 
(VA103A08 and GR105). J.S. holds an FPI (Formación de Personal Investigador) fellowship from the Spanish Ministerio de Educación y Ciencia. We thank the veterinaries of the slaughterhouse Justino Gutiérrez of Laguna de Duero (Valladolid) for providing cow adrenal glands.

\section{References}

Allersma, M.W., Wang, L., Axelrod, D., Holz, R.W., 2004. Visualization of regulated exocytosis with a granule-membrane probe using total internal reflection microscopy. Mol. Biol. Cell 15, 4658-4668.

Allersma, M.W., Bittner, M.A., Axelrod, D., Holz, R.W., 2006. Motion matters: secretory granule motion adjacent to the plasma membrane and exocytosis. Mol. Biol. Cell 17, 2424-2438.

Bezzi, P., Gundersen, V., Galbete, J.L., Seifert, G., Steinhäuser, C., Pilati, E., Volterra, A 2004. Astrocytes contain a vesicular compartment that is competent for regulated exocytosis of glutamate. Nat. Neurosci. 7, 613-620.

Bolte, S., Cordelières, F.P., 2006. A guided tour into subcellular colocalization analysis in light microscopy. J. Microsc. 224, 213-232.

Camacho, M., Machado, J.D., Montesinos, M.S., Criado, M., Borges, R., 2006 Intragranular $\mathrm{pH}$ rapidly modulates exocytosis in adrenal chromaffin cells. J. Neurochem. 96, 324-334.

Duman, J.G., Chen, L., Palmer, A.E., Hille, B., 2006. Contributions of intracellular compartments to calcium dynamics: implicating an acidic store. Traffic 7, 859872.

Duncan, R.R., Greaves, J., Wiegand, U.K., Matskevich, I., Bodammer, G., Apps, D.K., Shipston, M.J., Chow, R.H., 2003. Functional and spatial segregation of secretory vesicle pools according to vesicle age. Nature 422, 176-180.

Haller, T., Dietl, P., Deetjen, P., Völkl, H., 1996. The lysosomal compartment as intracellular calcium store in MDCK cells: a possible involvement in InsP3mediated $\mathrm{Ca}^{2+}$ release. Cell Calcium 19, 157-165.

Jadot, M., Biélande, V., Beauloye, V., Wattiaux-De Coninck, S., Wattiaux, R., 1990. Cytotoxicity and effect of glycyl-D-phenylalanine-2-naphthylamide on lysosomes. Biochim. Biophys. Acta 1027, 205-209.
Kuijpers, G.A., Rosario, L.M., Ornberg, R.L., 1989. Role of intracellular pH in secretion from adrenal medulla chromaffin cells. J. Biol. Chem. 264, 698-705.

Lang, T., Wacker, I., Steyer, J., Kaether, C., Wunderlich, I., Soldati, T., Gerdes, H.H., Almers, W., 1997. $\mathrm{Ca}^{2+}$-triggered peptide secretion in single cells imaged with green fluorescent protein and evanescent-wave microscopy. Neuron 18, 857863.

Li, D., Xiong, J., Qu, A., Xu, T., 2004. Three-dimensional tracking of single secretory granules in live PC12 cells. Biophys. J. 87, 1991-2001.

Manders, E., Stap, J., Brakenhoff, G., van Driel, R., Aten, J., 1992. Dynamics of threedimensional replication patterns during the S-phase, analyzed by double labelling of DNA and confocal microscopy. J. Cell Sci. 103, 857-862.

Moro, M.A., López, M.G., Gandía, L., Michelena, P., García, A.G., 1990. Separation and culture of living adrenaline- and noradrenaline-containing cells from bovine adrenal medullae. Anal.Biochem. 185, 243-248.

Nadrigny, F., Li, D., Kemnitz, K., Ropert, N., Koulakoff, A., Rudolph, S., Vitali, M. Giaume, C., Kirchhoff, F., Oheim, M., 2007. Systematic colocalization errors between acridine orange and EGFP in astrocyte vesicular organelles. Biophys. J. 93, 969-980.

Ohara-Imaizumi, M., Nakamichi, Y., Tanaka, T., Ishida, H., Nagamatsu, S., 2002 Imaging exocytosis of single insulin secretory granules with evanescent wave microscopy: distinct behavior of granule motion in biphasic insulin release. J. Biol. Chem. 277, 3805-3808.

Oheim, M., Stühmer, W., 2000. Interaction of secretory organelles with the membrane. J. Membr. Biol. 178, 163-173.

SantoDomingo, J., Vay, L., Camacho, M., Hernández-Sanmiguel, E., Fonteriz, R.I., Lobatón, C.D., Montero, M., Moreno, A., Alvarez, J., 2008. Calcium dynamics in bovine adrenal medulla chromaffin cell secretory granules. Eur. J. Neurosci. 28 1265-1274

Steyer, J.A., Horstmann, H., Almers, W., 1997. Transport, docking and exocytosis of single secretory granules in live chromaffin cells. Nature $388,474-478$.

Steyer, J.A., Almers, W., 1999. Tracking single secretory granules in live chromaffin cells by evanescent-field fluorescence microscopy. Biophys. J. 76, 2262-2271.

Straub, M., Lodemann, P., Holroyd, P., Jahn, R., Hell, S.W., 2000. Live cell imaging by multifocal multiphoton microscopy. Eur. J. Cell Biol. 79, 726-734.

Tsuboi, T., Zhao, C., Terakawa, S., Rutter, G.A., 2000. Simultaneous evanescent wave imaging of insulin vesicle membrane and cargo during a single exocytotic event Curr. Biol. 10, 1307-1310. 